\title{
REVIEW OF THE LONGITUDINAL IMPEDANCE BUDGET OF THE ELETTRA STORAGE RING
}

\author{
G. Penco*, C. Bontoiu, P. Craievich, E. Karantzoulis, V. Forchì \\ Sincrotrone Trieste, ELETTRA, Italy
}

\section{Abstract}

Changes in the longitudinal impedance budget occur due to the changes in the machine structure. Following the installation in ELETTRA storage ring of the new vacuum chambers in the last three years, estimation of the longitudinal loss factor are carried out. The measurements are performed by mapping the horizontal closed orbit deviation, taking full advantage of the newly installed high resolution BPM electron system. The current results are compared with those of the previous measurements and presented in this paper.

\section{INTRODUCTION}

ELETTRA is the Italian third generation synchrotron light source generating photons, mainly provided by insertion devices, from VUV to soft X-rays. The required high brilliance and quality of the photons are guaranteed by the stringent requirements on the electron beam parameters, its high stability, and the maximum achievable beam current. In general, impedances should be minimized to reduce the wakefields excited by the beam, which can influence the design parameters of the storage ring such as the energy spread as well as the effective emittance. The microwave longitudinal instability threshold is defined by the longitudinal impedance while the longitudinal loss factor, related with real part of the longitudinal impedance, provides the energy loss of a beam and defines the power released into the vacuum chamber by a train of bunches. Apart from the rf cavities which are four single cells, the other important contributor to the overall impedance is the vacuum chamber. Its contribution comes from both its shape and material as well as from its discontinuities such as holes, steps and tapers.

In reference [1] impedance budget measurements on the ELETTRA storage ring is extensively handled. Recently the Libera electron [2] device was used to upgrade the original Beam Position Monitor (BPM) detectors. These new detectors provide micrometric accuracy and fast data rate of the beam position measurements and thus the previous impedance budget has been updated. Measurements of the longitudinal loss factor are presented in this paper. Furthermore the issue of the thermal stabilization of the storage ring during and after the injection is taken into account in the measurements.

\footnotetext{
* giuseppe.penco@elettra.trieste.it
}

\section{PHYSICAL PRINCIPLE}

An electron beam passing through any irregularity of a vacuum chamber loses energy proportionally to the beam charge $Q_{b}$ and to the induced voltage in the structure $V_{\text {ind }}=-Z_{\|} I_{b}$, where $Z_{\|}$is the longitudinal impedance and $I_{b}$ is the beam current. Since $I_{b}$ is proportional to $Q_{b}$, the total energy loss of the beam $\left(E_{\text {loss }}\right)$ is proportional to the square of its charge, as [3]:

$$
E_{\text {loss }}=-k_{\|} Q_{b}^{2}
$$

The proportionality factor $k_{\|}$is the total longitudinal loss factor and it depends on the real part of $Z_{\|}$and on the Fourier transform $\tilde{\rho}(\omega)$ of the particle distribution $\rho(t)$ :

$$
k_{||}=\frac{1}{\pi} \int_{0}^{\infty} \Re Z_{||}|\rho(\tilde{\omega})|^{2} d \omega
$$

The measurement of $k_{\| \mid}$for the ELETTRA storage ring is presented in this paper.

Since the energy loss of equation 1 changes by varying the beam charge, an indirect method to measure $k_{\|}$is to take the closed orbit at a reference beam current $I_{0}$ and measuring the horizontal BPMs readings deviation in the dispersive region of the storage ring versus the beam current [1]:

$$
\Delta x(z) \cong \eta(z) \frac{\Delta E}{E_{b}}
$$

where $\eta(z)$ is the dispersion, $E_{b}$ is the total beam energy at the beam current $I_{b}$ and $\Delta E$ is the energy loss difference when the beam current changes from $I_{0}$ to $I_{b}$.

\section{EXPERIMENTAL RESULTS}

\section{Thermal Stabilization}

An important aspect to be treated during the measurement of the horizontal closed orbits is the thermal expansion of the vacuum chamber due to the synchrotron radiation emission. The power per turn converted into thermal energy varies linearly with the beam current and proportional to the fourth power of the energy as given by [4]:

$$
P[W]=8.85 \cdot 10^{4} \frac{E[G e V]^{4}}{R[m]} I_{\text {beam }}[A]
$$

with $\mathrm{R}$ the radius of the storage ring.

At ELETTRA ( $R \sim 41.25 m$ ), as soon as the beam energy is increased to $\mathrm{E}=2 \mathrm{GeV}$, heating of the vacuum chamber becomes noticeable for the BPM system: dissipated power along the whole ring reaches more than $10 \mathrm{~kW}$ at $300 \mathrm{~mA}$. 
The BPMs drift in an unsynchronized way due to the fact that they are installed in the vicinity of chambers of different geometries and materials, each of them with their own thermal expansion coefficients. Moreover during energy ramping from $0.9 \mathrm{GeV}$ to $2.0 \mathrm{GeV}$ the magnet yokes suffer from thermal dilations and therefore the closed orbit is distorted. Keeping the current constant, $\mathrm{I}=4.4 \mathrm{~mA}$, the rms value of the closed orbit was recorded during 16 mins from the moment when the beam was ramped. As shown in figure 1 the heating effects saturate after about 5 mins inducing a horizontal error of around $\Delta x_{r m s}=13 \mu \mathrm{m}$.

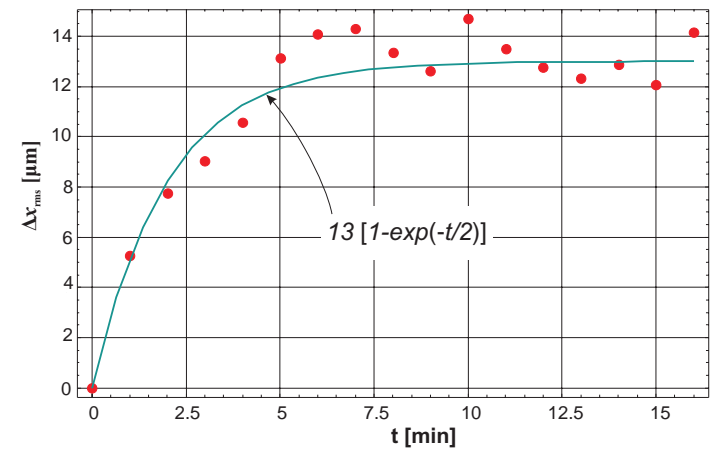

Figure 1: Rms values of the closed orbits as heating proceeds at constant beam current $\mathrm{I}=4.4 \mathrm{~mA}$.

In order to limit the thermal expansion, the closed orbit deviation versus beam current has been measured just at injection energy, $0.9 \mathrm{GeV}$. The heating due to the variation of the current is assumed to be less efficient than the energy variation (see eq. 4), introducing a negligible systematic error in the closed orbit deviation.

\section{Measurements}

In eq. 3 it has been assumed that the orbit deviation produced by the different $E_{\text {loss }}$ at different beam current is provided by the dispersion $\eta(z)$, neglecting eventual second order effects in the bending magnets. Figure 2 shows the measurements of the unoptimized horizontal dispersion at $0.9 \mathrm{GeV}$, after uniformly filling the storage ring (multibunch mode). Figure 3 shows the horizontal orbit deviation versus beam current. To increase the accuracy the orbit deviation values at the points with a dispersion higher than $10 \mathrm{~cm}$ have been taken in account to calculate the variation in the coherent energy loss, as:

$$
\Delta E=E_{0} Q_{b} \frac{\Delta x(z)}{\eta(z)}
$$

where $E_{0}=0.9 \mathrm{GeV}$ and $E_{0} \cdot Q_{b}=E_{b}$.

By quadratically fitting the energy loss difference versus the beam charge a longitudinal loss factor $k_{\|}=2.4 \pm$ $0.1 \mathrm{~V} / \mathrm{pC}$ is obtained in multi-bunch operation mode (see figure 4).

These results are quite different from what was measured at ELETTRA three years ago $\left(k_{\|}=12 \pm 2 \mathrm{~V} / p \mathrm{C}\right)$ mainly 05 Beam Dynamics and Electromagnetic Fields

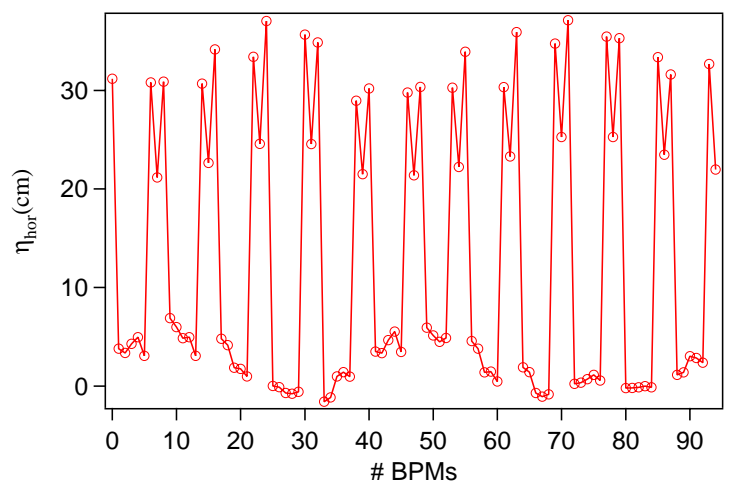

Figure 2: Unoptimized horizontal dispersion at $0.9 \mathrm{GeV}$, multi-bunch mode operation.

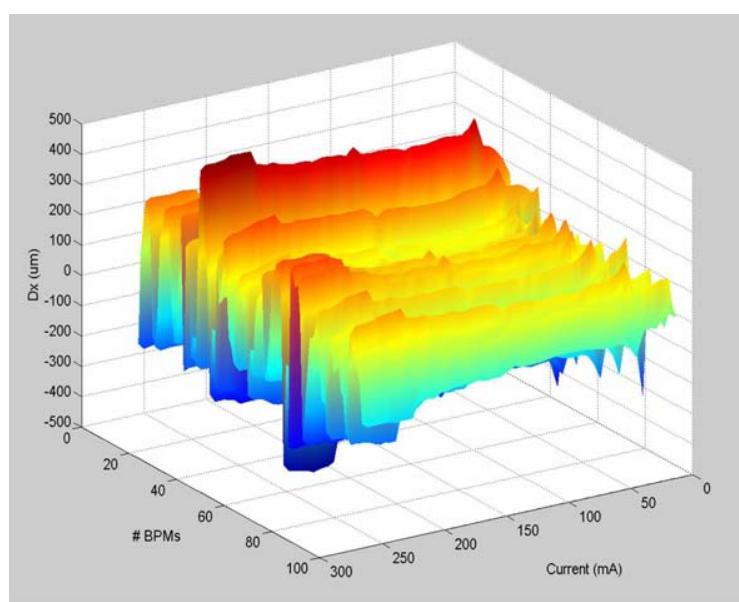

Figure 3: Horizontal closed orbit deviation versus beam current.

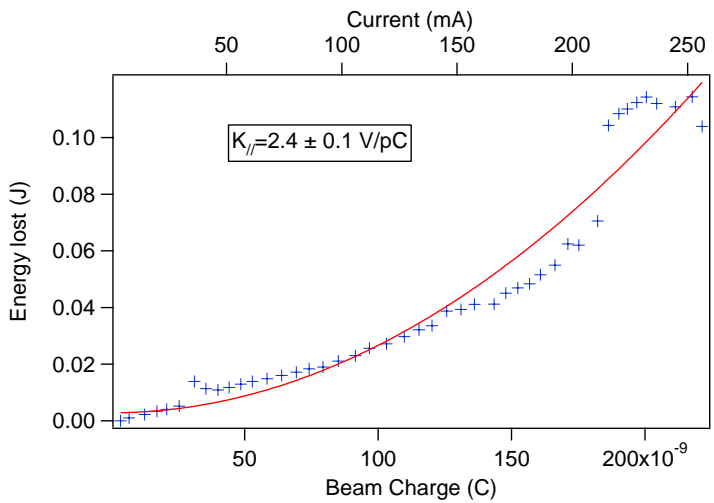

Figure 4: Energy loss difference versus beam charge (or beam current) fitting for the multi bunch mode operation at $0.9 \mathrm{GeV}$.

for the large improvement in the resolution of the present BPMs system, which provides a reading error two order of magnitude smaller than in the past ( $R M S<<0.1 \mu m[2])$.

A very roughly estimation of the main impedances sources at ELETTRA provides the following result, considering a RMS bunch length of $3 \mathrm{~mm}$ :

D04 Instabilities - Processes, Impedances, Countermeasures 
- $200 \mathrm{~m}$ circular vacuum chamber ( $\mathrm{r}=28 \mathrm{~mm}): 0.3 \mathrm{~V} / \mathrm{pC}$;

- $60 \mathrm{~m}$ elliptical Al chamber (gap=14mm): 0.07V/pC;

- the superconducting idle third harmonic cavity: $1.0 \mathrm{~V} / \mathrm{pC}$;

These calculations lead to a total $k_{\|}=1.37 \mathrm{~V} / \mathrm{pC}$, which is in good agreement with the measured value of $2.4 \mathrm{~V} / \mathrm{pC}$. In fact in the last calculation we have not included the several tapers between different transversal section of the vacuum chamber (for example the tapers at the exit of RF cavities).

By using the same experimental setup, measurements of $k_{\|}$have been repeated operating ELETTRA in few bunches. Figure 5 shows the results of the fitting for the 4-bunches case: a value of $15.8 \mathrm{~V} / \mathrm{pC}$ is obtained.

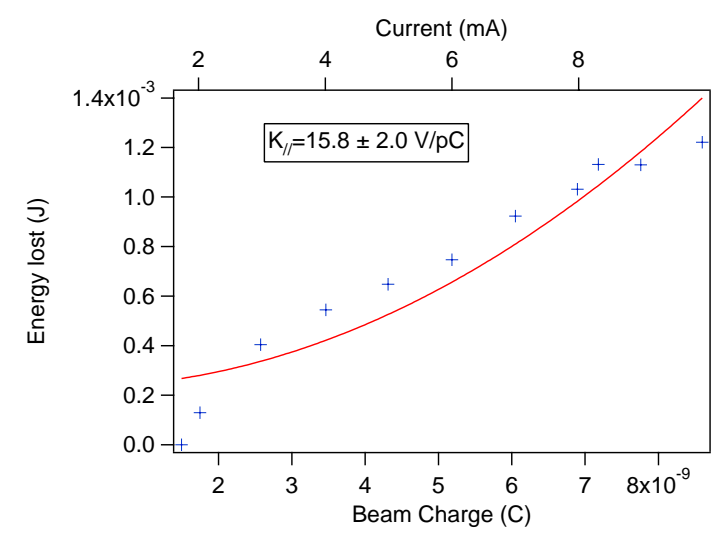

Figure 5: Fitting results of the energy loss difference versus beam charge for the 4 bunches case at $0.9 \mathrm{GeV}$.

In case of broad band impedances, the natural bunch lengthening induced by the bunch charge increment should lead to a reduction of the $k_{\|}$, according to the well-known equation [3]: $k_{\|} \propto 1 / \sigma^{3 / 2}$. Thus the measurements with few bunches, since the charge per bunch is higher, should give an inferior value with respect to the multibunch measurements. Nevertheless the observed increase of the loss factor when reducing the number of bunches circulating in the ring indicates that the impedances spectrum of ELETTRA could be dominated by localized narrow band impedances; in other words the spectral lines of the electron beam in few-bunches mode are comparable to the scale of variations of the machine impedance.

\section{CONCLUSION}

The longitudinal loss factor of ELETTRA storage ring has been measured by correlating the closed orbit deviation in the high dispersive points along the ring with the beam current increment. A very high accuracy of the new Liberas BPM system has improved the measurements resolution with respect to the past. Future experiments are going to be carried on to correlate the bunch length increase versus the bunch current in order to calculate the longitudinal impedance and compare it with these results.

\section{ACKNOWLEDGEMENTS}

The authors would like to thank M. Cornacchia and $\mathrm{S}$. Tazzari for discussions about related beam dynamics issues.

\section{REFERENCES}

[1] E. Karantzoulis, V. Smaluk and L.Tosi, "Broad band Impedance measurements on the electron storage ring ELETTRA”, Phys. Rev. ST-AB, Vol. 6, 030703 (2003).

[2] M. Lonza et al., "BPM Detectors Upgrade for the Elettra Fast Orbit Feedback”, DIPAC '07, Venice, May 2007.

[3] B.W. Zotter and S.A. Kheifets, "Impedances and Wakes in High-Energy Particle Accelerators”, World Scientific, 2000.

[4] J.D. Jackson, "Classical Electrodynamics", 2nd ed., John Wiley and Sons, Inc., 1975, p. 662. 